\title{
Differentiation-free Taylor approximation of finite motion in closed loop kinematics
}

\author{
J.J. de Jong ${ }^{1}$ A. Müller ${ }^{2}$ J. van Dijk ${ }^{1}$ J.L. Herder ${ }^{1}$ \\ ${ }^{1}$ University of Twente, The Netherlands, e-mail: j.j.de jong@utwente.nI, \\ ${ }^{2}$ Johannes Kepler University, Linz, Austria, e-mail: a. muel l er $a j k u$. at
}

\begin{abstract}
Higher-order derivatives of kinematic mappings give insight into the motion characteristics of complex mechanisms. Screw theory and its associated Lie group theory have been used to find these derivatives of loop closure equations up to an arbitrary order. However this has not been extended to the higher-order derivatives of finite motion as given by the inverse or forward kinematic model of closed loop mechanisms. In this paper, a recursive algorithm is presented, consisting solely of matrix multiplications, which is capable of giving these higher-order derivatives of kinematic models of closed loop linkages. It depends on a simplified representation of the higherorder derivatives of an open chain. From these higher-order derivatives a Taylor expansion of a finite motion becomes available. The evaluation of this method on a Taylor approximation (up to 5th order) of the inverse kinematic model of a 5-bar mechanism shows a good approximation in a large part of workspace around the evaluation point.
\end{abstract}

Key words: Higher-order kinematics, Taylor approximation, screw theory, 5-bar mechanism

\section{Introduction}

Geometric insight of infinitesimal motion of spatial kinematics can be obtained with the differential analysis of screw theory. This theory gives the kinematic relations between the general velocities of bodies (twists) and general constraint forces (constraint wrenches) acting on a system. This instantaneous analysis is only available in the pose of inspection, and in general does not give an understanding of the possible finite motion of a mechanism. For synthesis and analysis purposes, attempts have been made to extend the infinitesimal screw analysis. The finite forward kinematic model of open loop chains is given by Brockett's products of exponents (POE)[1]. It consists of the products of exponential matrices of the instantaneous screw axes. Derivatives up to an arbitrary order of loop closure equations can be found by taking Lie brackets of instantaneous screw axes, which can be expressed as matrix multiplications of twists[2,3]. This paves the way for an algorithmic differentiation-free derivatives of the loop closure equations [4].

However, higher-order derivatives and approximations of the finite motion of these closed loop mechanisms were not yet found. These higher-order derivatives 
of finite motion can be used for finding conditions for invariant properties of kinematics and dynamics such as required for balancing, synthesis and analysis of rigid body motions. Moreover, such an approximation is advantageous since closed form kinematic mappings are not always available for the more complex mechanisms. Unfortunately, processing these higher-order, multivariate derivatives require elaborate bookkeeping, as can be seen in the implementation of the higher-order chain rule, the Faa di Bruno's rule[5].

In this paper a simplified representation of the higher-order derivatives of the screw systems is presented which has a structure similar to the Brockett's POE. With Vetters method for managing higher order matrix derivatives [6] this enables us to obtain a recursive, differentiation-free algorithm for higher-order derivatives of the solution to the closure equations. Using the resulting higher-order closure Jacobians, a Taylor approximation of the closed loop kinematics is performed. The steps taken are exemplified with an approximation of the inverse kinematic model of a 5-bar mechanism.

Before we introduce the higher-order derivatives of the loop closure solution, the screw algebra theory is revisited and applied to an open chain. Based on this a simplified representation of higher-order derivatives of an open chain is presented (2.2). After this the loop closure equations and the matrix derivatives are revisited (2.3-2.4). Using these rules finally the algorithm for determining the higher-order derivatives and its Taylor expansion is presented (2.5) and its implementation shown for a 5-bar mechanism (2.6).

\section{Method}

\subsection{Concepts and notation}

In the notation of screw theory as used in this paper, a reference frame $\left(\psi_{i}\right)$ is associated to each rigid body $i$. Points in space $(\boldsymbol{a})$ can be expressed with respect to this reference frame (denoted with superscript $\boldsymbol{a}^{i}$ ). A change of reference frame follows from the homogeneous transformation matrix which consists of a rotation matrix $(\boldsymbol{R})$ and a translation vector $(\boldsymbol{o})$. In the homogeneous representation the $\boldsymbol{a}^{i}$-vector is appended with a 1 .

$$
\boldsymbol{a}^{j}=\boldsymbol{H}_{i}^{j} \boldsymbol{a}^{i} \quad \boldsymbol{H}_{i}^{j}=\left[\begin{array}{cc}
\boldsymbol{R}_{i}^{j} & \boldsymbol{o}_{i}^{j} \\
\mathbf{0} & 1
\end{array}\right] \quad \dot{\boldsymbol{H}}_{i}^{j}=\left[\begin{array}{cc}
{\left[\boldsymbol{\omega}_{i}^{j} \times\right]} & \boldsymbol{v}_{i}^{j} \\
\mathbf{0} & 0
\end{array}\right] \boldsymbol{H}_{i}^{j}=\left[\boldsymbol{t}_{i}^{j, j} \times\right] \boldsymbol{H}_{i}^{j}
$$

The time derivative of the transformation matrix is given by the twist $\left(t_{i}^{k, j}\right)$, th generalized velocity, of body $i$ with respect to body $j$ expressed in frame $k$. For clarity reasons the subscript and second superscript are omitted when possible. The twist is a vector containing the angular $(\boldsymbol{\omega})$ and translational $(\boldsymbol{v})$ velocity. The $[\boldsymbol{\omega} \times]$ denotes the skew symmetric matrix form of $\boldsymbol{\omega}$. The twist's frame of expression changes with 
the adjoint transformation matrices here denoted with $\operatorname{Ad}\left(\boldsymbol{H}_{i}^{j}\right)$.

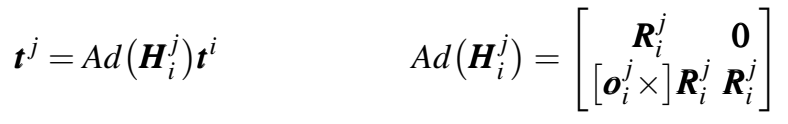

The time derivative of adjoint transformation matrix is given in terms of instantaneous transformation matrix $a d(\boldsymbol{t})$.

$$
\frac{d}{d t}\left(\operatorname{Ad}\left(\boldsymbol{H}_{i}^{j}\right)\right)=\operatorname{ad}\left(\boldsymbol{t}_{i}^{j, j}\right) \operatorname{Ad}\left(\boldsymbol{H}_{i}^{j}\right) \quad \operatorname{ad}(\boldsymbol{t})=\left[\begin{array}{cc}
{[\boldsymbol{\omega} \times} \\
{[\boldsymbol{v} \times]} & \mathbf{0} \\
{[\boldsymbol{\omega} \times]}
\end{array}\right]
$$

This matrix itself can be expressed in an other reference frame according to a nested transform:

$$
\operatorname{ad}\left(\boldsymbol{t}^{j}\right)=\operatorname{ad}\left(\operatorname{Ad}\left(\boldsymbol{H}_{i}^{j}\right) \boldsymbol{t}^{i}\right)=\operatorname{Ad}\left(\boldsymbol{H}_{i}^{j}\right) \operatorname{ad}\left(\boldsymbol{t}^{i}\right) \operatorname{Ad}\left(\boldsymbol{H}_{j}^{i}\right)
$$

Using these twists, a concise formulation for the forward kinematic mapping of an open chain is available in the form of Brockett's product of exponentials [1]:

$$
\operatorname{Ad}\left(\boldsymbol{H}_{n}^{0}(\boldsymbol{q})\right)=\prod_{i=1}^{n} \operatorname{Ad}\left(\boldsymbol{H}_{i}^{i-1}\left(q_{i}\right)\right)=\prod_{i=1}^{n} e^{a d\left(\hat{\boldsymbol{t}}_{i}^{0}\right) q_{i}} \operatorname{Ad}\left(\boldsymbol{H}_{n}^{0}(0)\right)
$$

In here, the instantaneous screw vector $\hat{\boldsymbol{t}}_{i}^{0}$, denoted with a hat, specifies the amount of twist generated by the instantaneous motion of joint $i$, and is therefore a pure geometric entity. As this screw vector is always with respect to the previous body in the chain, the second superscript is omitted. The instantaneous screw vector of lower kinematic pairs are constant when expressed in the connecting frames e.q. $\frac{d}{d t}\left(\hat{\boldsymbol{t}}_{i}^{i-1}\right)=\frac{d}{d t}\left(\hat{\boldsymbol{t}}_{i}^{i}\right)=\mathbf{0}$.

\subsection{Derivatives of twist systems (open chain)}

For an open chain, the higher-order partial derivatives can be found using the transformations of the previous section. A chain of transformations can be decomposed into constant and varying part of which the derivative is available. The nested transform (4) of the twist gives a concise formulation of the derivative of a chain, provided that $i \leq n$.

$$
\begin{aligned}
\frac{d}{d q_{i}}\left(\operatorname{Ad}\left(\boldsymbol{H}_{n}^{0}\right)\right) & =\operatorname{Ad}\left(\boldsymbol{H}_{i-1}^{0}\right) \frac{d}{d q_{i}}\left(\operatorname{Ad}\left(\boldsymbol{H}_{i}^{i-1}\right)\right) \operatorname{Ad}\left(\boldsymbol{H}_{n}^{i}\right) \\
& =\operatorname{Ad}\left(\boldsymbol{H}_{i-1}^{0}\right) \operatorname{ad}\left(\hat{\boldsymbol{t}}_{i}^{i-1}\right) \operatorname{Ad}\left(\boldsymbol{H}_{i}^{i-1}\right) \operatorname{Ad}\left(\boldsymbol{H}_{n}^{i}\right) \\
& =\operatorname{ad}\left(\hat{\boldsymbol{t}}_{i}^{0}\right) \operatorname{Ad}\left(\boldsymbol{H}_{n}^{0}\right)
\end{aligned}
$$


For the second-order, such a concise representation also exists. For the consecutive derivative with respect to joint $j$ there exist two possibilities, either it is after body $i$ in the chain (case 1.) or before $i$ in the chain (case 2).

1. Case 1. $(i \leq j)$ In the case that joint $j$ is higher in the chain than $i$, the twist is unaffected $\left(\frac{d}{d q_{j}}\left(\operatorname{ad}\left(\hat{\boldsymbol{t}}_{i}^{0}\right)\right)=0\right)$. Therefore, the second partial derivative becomes:

$$
\frac{d}{d q_{j}} \frac{d}{d q_{i}}\left(\operatorname{Ad}\left(\boldsymbol{H}_{n}^{0}\right)\right)=\operatorname{ad}\left(\hat{\boldsymbol{t}}_{i}^{0}\right) \operatorname{ad}\left(\hat{\boldsymbol{t}}_{j}^{0}\right) \operatorname{Ad}\left(\boldsymbol{H}_{n}^{0}\right)
$$

2. Case 2. $(i \geq j)$ In the case that $j$ is below $i$ in the chain we use the nested transform property to split the chain into a dependent and independent part. It may be verified that $\frac{d}{d q_{j}}\left(\operatorname{ad}\left(\hat{\boldsymbol{t}}_{i}^{j}\right) \operatorname{Ad}\left(\boldsymbol{H}_{n}^{j}\right)\right)=0$. Therefore:

$$
\begin{aligned}
\frac{d}{d q_{j}} \frac{d}{d q_{i}}\left(\operatorname{Ad}\left(\boldsymbol{H}_{n}^{0}\right)\right) & =\frac{d}{d q_{j}}\left(\operatorname{Ad}\left(\boldsymbol{H}_{j}^{0}\right) \operatorname{ad}\left(\hat{\boldsymbol{t}}_{i}^{j}\right) \operatorname{Ad}\left(\boldsymbol{H}_{n}^{j}\right)\right) \\
& =\frac{d}{d q_{j}}\left(\operatorname{Ad}\left(\boldsymbol{H}_{j}^{0}\right)\right) \operatorname{ad}\left(\hat{\boldsymbol{t}}_{i}^{j}\right) \operatorname{Ad}\left(\boldsymbol{H}_{n}^{j}\right)
\end{aligned}
$$

Using (8) a matrix chain can be found and collected again using the nested transform:

$$
\frac{d}{d q_{j}} \frac{d}{d q_{i}}\left(\operatorname{Ad}\left(\boldsymbol{H}_{n}^{0}\right)\right)=\operatorname{ad}\left(\hat{\boldsymbol{t}}_{j}^{0}\right) \operatorname{ad}\left(\hat{\boldsymbol{t}}_{i}^{0}\right) \operatorname{Ad}\left(\boldsymbol{H}_{n}^{0}\right)
$$

Leaves us with an expression similar to (9), with the difference that sequence of multiplication is swapped. This also follows from the symmetry (commutativity) property of mixed partial derivatives: $\frac{d}{d q_{j}} \frac{d}{d q_{i}}\left(\operatorname{Ad}\left(\boldsymbol{H}_{n}^{0}\right)\right)=\frac{d}{d q_{i}} \frac{d}{d q_{j}}\left(\operatorname{Ad}\left(\boldsymbol{H}_{n}^{0}\right)\right)$.

A consecutive application of (9), and (12) gives us the geometrical higher-order partial derivatives for any order, supplied in multi-index $\boldsymbol{\alpha}^{1}$, which is ordered from the base to the end-effector.

$$
D_{\boldsymbol{q}}^{(\boldsymbol{\alpha})}\left(\operatorname{Ad}\left(\boldsymbol{H}_{n}^{0}\right)\right)=\prod_{i=1}^{n}\left(\left(\operatorname{ad}\left(\hat{\boldsymbol{t}}_{i}^{0}\right)\right)^{\boldsymbol{\alpha}(i)}\right) \operatorname{Ad}\left(\boldsymbol{H}_{n}^{0}\right)
$$

This result is similar to that of [3], with the difference that the index ranges to distinguish between the sequence of derivatives are taken into account by the ordering of $\boldsymbol{\alpha}$. From the commutative property of mixed partial derivatives it follows that for whatever sequence of differentiation the same results are obtained. Furthermore, it can be seen that (13) resembles the structure of the Brockett's formula (5) in the sense that the matrix multiplications follow the physical ordering of the chain.

${ }^{1} D_{\boldsymbol{x}}^{(k)}(\boldsymbol{A})$ denotes the matrix collection of all $k$-th order partial derivatives of $\boldsymbol{A}$ with respect to $\boldsymbol{x} . D_{\boldsymbol{x}}^{(\boldsymbol{\alpha})}(\boldsymbol{A})$ denotes the mixed partial derivative with respect to the elements of $\boldsymbol{x}$. A sequence of derivatives to each $\boldsymbol{x}_{i}$ with an order of the corresponding $\boldsymbol{\alpha}_{i}$ value. This assumes that the mixed partial derivative are commutative. 


\subsection{Loop closure equations}

The open-loop chain derivatives (13) can be used for closed loops, as a closed loop can be seen as a connection of multiple open-loops. E.g., a simple loop can be seen as a open chain of with the last link fixed to the base. The loop closure equation $(f)$ states how the members of the loop are constrained. It can be written in terms of independent $(\boldsymbol{u})$ and dependent coordinates $(\boldsymbol{v})$, also termed input and output, respectively. The total set of coordinates we call $\boldsymbol{s}^{\top}=\left[\boldsymbol{u}^{\top} \boldsymbol{v}^{\top}\right]$. The solution to this problem is denoted by $c$, which can be the inverse, forward, or any other kinematic model giving an exact relation between independent and dependent coordinates.

$$
f(\boldsymbol{u}, \boldsymbol{v})=\mathbf{0}=c(\boldsymbol{u})
$$

The solution $(c)$ to the loop closure is usually not available for complex mechanisms. Therefore, we are looking for a Taylor expansion using higher-order derivatives of the constraint formulation using the open loop derivatives of section 2.2. We start with the first order. This reads:

$$
\mathbf{0}=D_{t}(f)=D_{\boldsymbol{u}}(f) \dot{\boldsymbol{u}}+D_{\boldsymbol{v}}(f) \dot{\boldsymbol{v}}=\boldsymbol{U} \dot{\boldsymbol{u}}+\boldsymbol{V} \dot{\boldsymbol{v}}
$$

This gives rise to the Jacobians $(\boldsymbol{C})$ and $(\boldsymbol{K})$, respectively linking $\dot{\boldsymbol{v}}$ and $\dot{\boldsymbol{w}}$ to $\dot{\boldsymbol{u}}$.

$$
\dot{\boldsymbol{v}}=-\boldsymbol{V}^{-1} \boldsymbol{U} \dot{\boldsymbol{u}}=\boldsymbol{C} \dot{\boldsymbol{u}}=D_{\boldsymbol{u}}(c) \dot{\boldsymbol{u}} \quad \dot{\boldsymbol{s}}=\boldsymbol{K} \dot{\boldsymbol{u}}=\left[\begin{array}{c}
\boldsymbol{C} \\
\boldsymbol{I}
\end{array}\right] \dot{\boldsymbol{u}}
$$

We already have seen that closure equations can be written as a function of transformation matrices of the open chain. Therefore, the higher-order partial derivatives of $D_{\boldsymbol{s}}^{(\boldsymbol{\alpha})}(\boldsymbol{U})$ and $D_{\boldsymbol{s}}^{(\boldsymbol{\alpha})}(\boldsymbol{V})$ are available. Now we are looking for a method of writing the higher-order closure Jacobian $\boldsymbol{C}_{k}=D_{\boldsymbol{u}}^{(k)}(c)$

\subsection{Multivariate matrix derivatives using Kronecker product}

The higher-order partial derivatives of matrices can be managed with the use of the Kronecker product [6]. Here the partial derivative version of the product rule, the chain rule and the inverse matrix derivative are given.

- Product rule of $\boldsymbol{A}(\boldsymbol{x}) \in \mathbb{R}^{n \times m}, \boldsymbol{B}(\boldsymbol{x}) \in \mathbb{R}^{m \times q}$, for $\boldsymbol{x} \in \mathbb{R}^{r}$, and $\boldsymbol{I}$ is an identity matrix:

$$
\begin{aligned}
D_{\boldsymbol{x}}(\boldsymbol{A} \boldsymbol{B}) & =\left[D_{\boldsymbol{x}}\left(\boldsymbol{a}_{1}\right) \boldsymbol{B} \ldots D_{\boldsymbol{x}}\left(\boldsymbol{a}_{m}\right) \boldsymbol{B}\right]+\boldsymbol{A} D_{\boldsymbol{x}}(\boldsymbol{B}) \\
& =D_{\boldsymbol{x}}(\boldsymbol{A})\left(\boldsymbol{B} \otimes \boldsymbol{I}_{r}\right)+\boldsymbol{A} D_{\boldsymbol{x}}(\boldsymbol{B})
\end{aligned}
$$

- Chain rule:

$$
D_{\boldsymbol{c}}(\boldsymbol{A}(\boldsymbol{b}(\boldsymbol{c})))=D_{\boldsymbol{b}}(\boldsymbol{A})\left(\boldsymbol{I}_{m} \otimes D_{\boldsymbol{c}}(\boldsymbol{b})\right)
$$

- Derivative of matrix inversion: 


$$
D_{\boldsymbol{x}}\left(\boldsymbol{A}^{-1}\right)=-\boldsymbol{A}^{-1} D_{\boldsymbol{x}}(\boldsymbol{A})\left(\boldsymbol{A}^{-1} \otimes \boldsymbol{I}_{r}\right)
$$

Recursive applications of these rules allow the extension of these derivatives to higher orders.

\subsection{Higher-order closure Jacobians and Taylor approximation}

Using the rules of the previous section, the second-order derivatives (Hessian) of the solution to the constraint equations are found. This is done by consecutive application of the chain rule, the product rule, and the inverse matrix derivative to the Jacobian (16) .

$$
\begin{aligned}
D_{\boldsymbol{u}}\left(\boldsymbol{C}_{1}\right)=\boldsymbol{C}_{2} & =-\left[D_{\boldsymbol{s}}\left(\boldsymbol{V}^{-1}\right)(\boldsymbol{U} \otimes \boldsymbol{I})-\boldsymbol{V}^{-1} D_{\boldsymbol{s}}(\boldsymbol{U})\right](\boldsymbol{I} \otimes \boldsymbol{K}) \\
& =-\boldsymbol{V}^{-1}\left[D_{\boldsymbol{s}}(\boldsymbol{V})\left(\boldsymbol{C}_{1} \otimes \boldsymbol{I}\right)+D_{\boldsymbol{s}}(\boldsymbol{U})\right](\boldsymbol{I} \otimes \boldsymbol{K})
\end{aligned}
$$

After reordering and combination of the Kronecker products, we can find a concise formulation of the Hessian matrix.

$$
\boldsymbol{C}_{2}=-\boldsymbol{V}^{-1}\left[D_{\boldsymbol{s}}(\boldsymbol{V}) D_{\boldsymbol{s}}(\boldsymbol{U})\right](\boldsymbol{K} \otimes \boldsymbol{K})=-\boldsymbol{V}^{-1} \boldsymbol{F}_{2} \boldsymbol{G}_{2}
$$

A further derivation is applied to show that a similar structure as the Hessian can be found for the 3 rd derivative. For higher orders this process can be repeated until the desired order is reached, giving us a recursive algorithm.

$$
D_{\boldsymbol{u}}\left(\boldsymbol{C}_{2}\right)=-\boldsymbol{V}^{-1}\left[D_{\boldsymbol{s}}(\boldsymbol{V}) D_{\boldsymbol{s}}\left(\boldsymbol{F}_{2}\right) \boldsymbol{F}_{2}\right]\left[\begin{array}{c}
\boldsymbol{C}_{2} \otimes \boldsymbol{K} \\
\boldsymbol{G}_{2} \otimes \boldsymbol{K} \\
D_{\boldsymbol{u}}\left(\boldsymbol{G}_{2}\right)
\end{array}\right]=-\boldsymbol{V}^{-1} \boldsymbol{F}_{3} \boldsymbol{G}_{3}
$$

This algorithm consist of three steps: 1) The higher-order derivatives of $\boldsymbol{V}$, and $\boldsymbol{U}$ are filled into the proper location of $\boldsymbol{F}_{k}$. These can be found a priori by higherorder screw derivatives of the open-loop equivalent. 2) The $\boldsymbol{G}_{k}$ matrix is filled with precursory, lower-order results. 3) The combination of the three matrices give the subsequent partial derivative of the closure Jacobian $\left(\boldsymbol{C}_{k}\right)$. The derivatives of the $\boldsymbol{G}_{k}$ matrix involves permutation for the derivatives of the Kronecker product [6]. The exact nature of this permutation is outside the scope of this paper.

The Taylor approximation of the loop closure solution can now be written using the partial derivatives of the closure Jacobians up to the $k$-th order. We assume that at the evaluation point the closure constraint is satisfied, and that $\boldsymbol{s}=\mathbf{0}$ such that the Taylor series becomes a Maclaurin series. The input for the independent variables is given as a power (denoted with the ${ }^{\otimes i}$ ) of Kronecker products [6]:

$$
\boldsymbol{v}(\boldsymbol{u})=\mathbf{0}+\boldsymbol{C}_{1} \boldsymbol{u}+\frac{1}{2 !} \boldsymbol{C}_{2}(\boldsymbol{u} \otimes \boldsymbol{u})+\frac{1}{3 !} \boldsymbol{C}_{3}(\boldsymbol{u} \otimes \boldsymbol{u} \otimes \boldsymbol{u})+\ldots \approx \sum_{i=1}^{k} \frac{1}{i !} \boldsymbol{C}_{i} \boldsymbol{u}^{\otimes i}
$$




\subsection{Approximate solution of a 5-bar mechanism}

The higher-order derivatives and Taylor expansion is applied to approximate the inverse kinematic model of a 5-bar mechanism. We choose to describe the 5-bar as a connection of two open chains ( $a$, and $b$ ) with joints $q_{1}, q_{2}$ and $q_{3}, q_{4}$ respectively. The connection point is the end-effector $\boldsymbol{x}^{0}$. This point has to satisfy the constraint equation from both sides $(a, b)$ calculated using the connection point in the local frame ( $x^{2}$ and $\left.x^{4}\right)$. The closure equation can be written as:

$$
\boldsymbol{x}_{a}^{0}=\boldsymbol{H}_{2}^{0}\left(\boldsymbol{q}_{1,2}\right) \boldsymbol{x}^{2} \quad \boldsymbol{x}_{b}^{0}=\boldsymbol{H}_{4}^{0}\left(\boldsymbol{q}_{3,4}\right) \boldsymbol{x}^{4} \quad f: \mathbf{0}=\left[\begin{array}{l}
\boldsymbol{x}^{0}-\boldsymbol{x}_{a}^{0} \\
\boldsymbol{x}^{0}-\boldsymbol{x}_{b}^{0}
\end{array}\right]
$$

Using the end-effector coordinates $\left(\boldsymbol{u}=\boldsymbol{x}^{0}\right)$ as input and the 4 joint angles $(\boldsymbol{v}=$ $\left.\left[\begin{array}{lll}q_{1} & \ldots & q_{4}\end{array}\right]^{\top}\right)$ as output, the first-order partial derivatives of the closure equation become:

$$
D_{\boldsymbol{u}}(f)=\boldsymbol{U}=\left[\begin{array}{l}
\boldsymbol{I} \\
\boldsymbol{I}
\end{array}\right] \quad D_{\boldsymbol{v}}(f)=\boldsymbol{V}=\left[\begin{array}{cccc}
{\left[\hat{\boldsymbol{t}}_{1}^{0} \times\right] \boldsymbol{x}_{a}^{0}} & {\left[\hat{\boldsymbol{t}}_{2}^{0} \times\right] \boldsymbol{x}_{a}^{0}} & \mathbf{0} & \mathbf{0} \\
\mathbf{0} & \mathbf{0} & {\left[\hat{\boldsymbol{t}}_{3}^{0} \times\right] \boldsymbol{x}_{b}^{0}} & {\left[\hat{\boldsymbol{t}}_{4}^{0} \times\right] \boldsymbol{x}_{b}^{0}}
\end{array}\right]
$$

The higher-order partial derivatives can be found by using the twist derivatives of 2.2 and recursive equations of 2.5 .

\section{Results}

The Taylor approximation, up to the 5-th order, is done for 200 positions of the end-effector $\left(\boldsymbol{x}^{0}\right)$ forming 4 trajectories through the workspace with the aim to find an approximation of the corresponding joint displacement of the joints $\left(q_{1} \ldots q_{4}\right)$. For evaluation of the quality of the Taylor approximation, the end-effector position approximation from the left $\left(\boldsymbol{x}_{a}^{0}\right)$ and right $\left(\boldsymbol{x}_{b}^{0}\right)$ side are plotted together with input trajectories.

The result of the Taylor approximation (Figure 1) shows that in a large portion of the workspace around the evaluation point $\left(x^{0}=0\right)$ the approximation converges to input indicating a correct estimation of finite joint displacement. However, further from the evaluation point the accuracy is less as can be seen in the insert.

\section{Discussion and conclusion}

For the calculation of higher-order partial derivatives, this method uses Kronecker products of matrices, which can lead to very large matrices for larger systems and higher orders. This possibly poses practical limits on applicability of this procedure. Sparse matrices and the aggregation of mixed partial derivatives can be used to mit- 


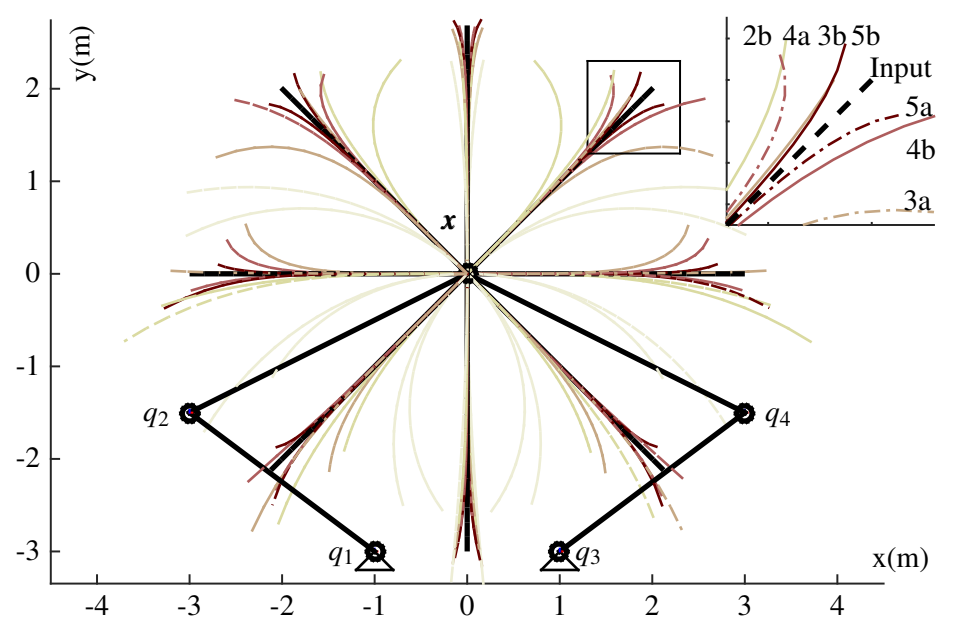

Fig. 1 The Taylor approximation of the IKM of a 5-bar (solid black) around evaluation point at $\boldsymbol{x}^{0}=[0,0]$ up to the 5 th order for 4 different trajectories. It shows the left (solid colored) and right (dashed colored) estimation of end-effector trajectory (dashed black). The insert shows convergence for higher-order estimation far from the evaluation point.

igate the memory usage and reduce the number of matrix operations. It is worth investigating what determines the validity of the Taylor approximations in kinematics, such as the radius of convergence and the closeness to singularities.

In this paper, a recursive method was presented which gives the higher-order partial derivatives of closure Jacobians of open and closed loop mechanisms consisting of lower kinematic pairs. This method relied on a simplified representation of the higher-order twist derivatives, also presented here, and the matrix derivatives of Vetter [6]. This enabled the Taylor approximation of a kinematic mapping over a given trajectory, as exemplified the 5-bar mechanism.

\section{References}

1. R W Brockett. Robotic manipulators and the product of exponentials formula. Mathematical Theory of Networks and Systems SE - 9, 58:120-129, 1984.

2. J.M. Rico, J Gallardo, and J. Duffy. Screw theory and higher order kinematic analysis of open serial and closed chains. Mechanism and Machine Theory, 34(4):559-586, 1999.

3. Andreas Müller. Higher derivatives of the kinematic mapping and some applications. Mechanism and Machine Theory, 76:70-85, jun 2014.

4. Andreas Müller. Recursive higher-order constraints for linkages with lower kinematic pairs. Mechanism and Machine Theory, 100(April):33-43, jun 2016.

5. Tsoy W. Ma. Higher chain formula proved by combinatorics. Electronic Journal of Combinatorics, 16(1):1-7, 2009.

6. William J. Vetter. Matrix Calculus Operations and Taylor Expansions. Society, 15(2):352-369, 2011. 